\title{
Continuous Learning of Compiler Heuristics
}

\author{
MICHELE TARTARA and STEFANO CRESPI REGHIZZI, Politecnico di Milano
}

\begin{abstract}
Optimizing programs to exploit the underlying hardware architecture is an important task. Much research has been done on enabling compilers to find the best set of code optimizations that can build the fastest and less resource-hungry executable for a given program. A common approach is iterative compilation, sometimes enriched by machine learning techniques. This provides good results, but requires extremely long compilation times and an initial training phase lasting even for days or weeks.

We present long-term learning, a new algorithm that allows the compiler user to improve the performance of compiled programs with reduced compilation times with respect to iterative compilation, and without an initial training phase. Our algorithm does not just build good programs: it acquires knowledge every time a program is compiled and it uses such knowledge to learn compiler heuristics, without the need for an expert to manually define them. The heuristics are evolved during every compilation, by evaluating their effect on the generated programs. We present implementations of long-term learning on top of two different compilers, and experimental data gathered on multiple hardware configurations showing its effectiveness.
\end{abstract}

Categories and Subject Descriptors: D.3.4 [Programming Languages]: Processors—Compilers; I.2.2 [Artificial Intelligence]: Automatic Programming-Program transformation

General Terms: Algorithms, Performance

Additional Key Words and Phrases: Iterative compilation, machine learning, PetaBricks

\section{ACM Reference Format:}

Tartara, M. and Crespi Reghizzi, S. 2013. Continuous learning of compiler heuristics. ACM Trans. Architec. Code Optim. 9, 4, Article 46 (January 2013), 25 pages.

DOI $=10.1145 / 2400682.2400705$ http://doi.acm.org/10.1145/2400682.2400705

\section{INTRODUCTION}

Building an exact model of modern computer architectures that a compiler can use to decide what optimizations algorithms to apply and what values to assign to her numerical parameters is impractical. Therefore, all compilers use heuristics to make such decisions, based on program-dependent values, called features.

Usually, compiler experts write the heuristics. This is a time-consuming task, and it is likely to yield suboptimal results since it is entirely based on the skill of one or few experts. Furthermore, in order to obtain the best results, the process of defining the heuristics should be repeated for every new architecture the compiler has to target.

In order to face these issues, researchers proposed various techniques. Iterative compilation [Kisuki et al. 2000] is the simplest one. It is based on generating hundreds of versions of the program being compiled, then running them while gathering execution times. The fastest version is then kept as the final result of the compilation. The

M. Tartara was partially supported by a Roberto Rocca Doctoral Fellowship, awarded by "Fondazione Fratelli Agostino ed Enrico Rocca" Lugano, CH.

Authors' address: M. Tartara (corresponding author), and S. Crespi Reghizzi, Dipartimento di Elettronica e Infomazione, Politecnico di Milano, Italy; email: mtartara@elet.polimi.it.

Permission to make digital or hard copies of part or all of this work for personal or classroom use is granted without fee provided that copies are not made or distributed for profit or commercial advantage and that copies show this notice on the first page or initial screen of a display along with the full citation. Copyrights for components of this work owned by others than ACM must be honored. Abstracting with credit is permitted. To copy otherwise, to republish, to post on servers, to redistribute to lists, or to use any component of this work in other works requires prior specific permission and/or a fee. Permissions may be requested from Publications Dept., ACM, Inc., 2 Penn Plaza, Suite 701, New York, NY 10121-0701 USA, fax +1 (212) 869-0481, or permissions@acm.org.

(c) 2013 ACM 1544-3566/2013/01-ART46 $\$ 15.00$

DOI 10.1145/2400682.2400705 http://doi.acm.org/10.1145/2400682.2400705 
various versions are created by applying a different compiler configuration for each of them. Depending on the specific iterative compilation algorithm considered, each configuration can determine the set of optimizations to be used, the order in which the optimization algorithms are applied, or some compilation parameters (e.g., loop unrolling factor, size of the tiles for a matrix tiling algorithm, limits for function inlining, etc.). The choice of which configurations, to try is determined by random selection. The overhead introduced by iterative compilation is too high for most applications, but the technique is useful in some specific areas, such as embedded systems [Bodin et al. 1998], where the available resources are scarce and a program is likely to be deployed on many identical systems, making the extra compilation time worth spending.

Machine learning compilation techniques have been introduced to restrict the configurations' search space [Kulkarni et al. 2004; Park et al. 2011]: a training phase is performed when the compiler is deployed, gathering data that are used to train a model. Whenever a new program needs to be compiled, the model is queried to predict good configurations. Usually, this just aims at focusing the selection of the configurations to be tested through iterative compilation on more promising areas [Agakov et al. 2006], but a similar approach can be used to predict a single configuration to be used as the result of the compilation [Fursin and Temam 2010]. Unfortunately, the training phase for building a good model is really long, up to several weeks [Fursin et al. 2008], and this limits the applicability of machine learning approaches.

The contributions of this article are as follows.

-We introduce long-term learning, a new learning algorithm for compilers able to build a model of the target system while compiling programs. The model is used to optimize the programs while avoiding both the long compilation times of iterative compilation and the initial training phase of machine-learning-based approaches.

-We show that, starting without knowledge of the target system, long-term learning is able to create heuristics at least as good as those of existing compilers (-03 for GCC and the default optimization level for PetaBricks). We also show that, starting from such optimization levels, our algorithm is able to improve them.

-We show that long-term learning is able to continuously improve the performance of a compiler online ${ }^{1}$, whereas most compiler learning algorithms work offline and require a long initial training phase.

-We show that different compilers can benefit from the use of long-term learning, by presenting two implementation and experimental results gathered on multiple hardware configurations.

The rest of this article is organized as follows: Section 2 describes the long-term learning compilation algorithm, Section 3 describes our implementations of the algorithm and the setup of the experimental campaign, Section 4 presents the results of the experiments. Related work is presented in Section 5 and possible future improvements are discussed in Section 6. Section 7 concludes.

\section{CONTINUOUS LEARNING COMPILATION}

Long-term learning is a new continuous learning evolutionary algorithm meant to avoid the main drawbacks of both iterative and machine-learning-based compilation: it only needs a small number of compilations and test runs to be performed, thus reducing compilation times with respect to iterative compilation; at the same time, it does not need the initial training phase usually required by machine learning algorithms.

\footnotetext{
${ }^{1}$ In this article online and offline are used with the meaning they have in the machine learning research community [Blum 1996]: the learning process is said to happen online when there are no distinct phases of learning and applying the learned knowledge. If the two phases are separated, the learning is termed offline.
} 
The aim of long-term learning is to learn a model of the target architecture, in the form of a set of heuristics that will allow the compiler to produce highly optimized programs without manual tuning by an expert. This learning process takes place over time: every time a new program is compiled, the system learns some new piece of information to be reused during the next compilations. This is possible thanks to the testing phase of the various candidate versions of the program, that happens as a part of the compilation process.

Long-term learning (detailed in Algorithm 1) is an evolutionary algorithm [Fogel 2000]. Each set of compiler heuristics (also termed candidate compiler configuration) is seen as an individual of a population. The population evolves along a given number of generations, each composed by the same number of individuals. The individuals of each generation derive from the best ones of the previous generation by modifying them slightly through a process named evolution (described in Section 2.1). At the end of each generation, a fitness function evaluates each individual according to the method explained in Section 2.6: the fittest individuals are then used as the seed to generate the candidates of the next generation.

In order to prevent stagnation of the process into local performance maxima, evolutionary algorithms define a mutation operation (detailed in Section 2.2), that modifies the candidate configurations more deeply than evolution, allowing to look for solutions that are far away from the current best ones. To speed up the convergence of evolutionary algorithms and to improve the stability of the solution found, a technique known as elitism (described in Section 2.4) can be used.

In our system, we decided to represent each compiler heuristic contained in the candidate configurations as a mathematical formula. The result value of the formula is the decision made by the heuristic, and variables of the formula represent features of the program being compiled. A feature is a value known by the compiler at compile time that describes a characteristic of the program (e.g., number of basic blocks in a function, loop nesting level, etc.). A similar representation of heuristics has been used by Stephenson et al. [2003], although for a system based on offline learning. In particular, we use static features: features that can be extracted by source-code analysis, without requiring the program to be executed.

The heuristics are not hard-coded into the compiler source code, to allow them to evolve easily: when the compiler needs to make a decision, it loads the current best heuristic for that decision from a knowledge base, evaluates it using the values of the features, and uses the obtained result as the outcome of the decision process.

In most evolutionary algorithms the individuals of each generation descend from the individuals of the previous generation only. Not so in long-term learning: at the end of every generation, all the available information is stored in a knowledge base (see Section 2.5), and the new candidates are evolved from the best ones of the whole knowledge base. Moreover, we do not just store information about the best candidates, but about all of them, together with their fitness level describing how good each heuristic has proved to be over the whole life of the compiler. We can thus reduce the number of generations needed to obtain good candidates, since we are using all the already gathered data instead of partial information. The heuristics stored in the knowledge base (and in particular, the best-scoring ones) constitute the model of the target system that the algorithm learns over time.

The general workflow of a compiler using long-term learning is represented in Figure 1.

\subsection{Evolution}

In order to learn, every evolutionary algorithm needs to explore the solution space by generating and testing new candidates. As the name "evolution" suggests, new 


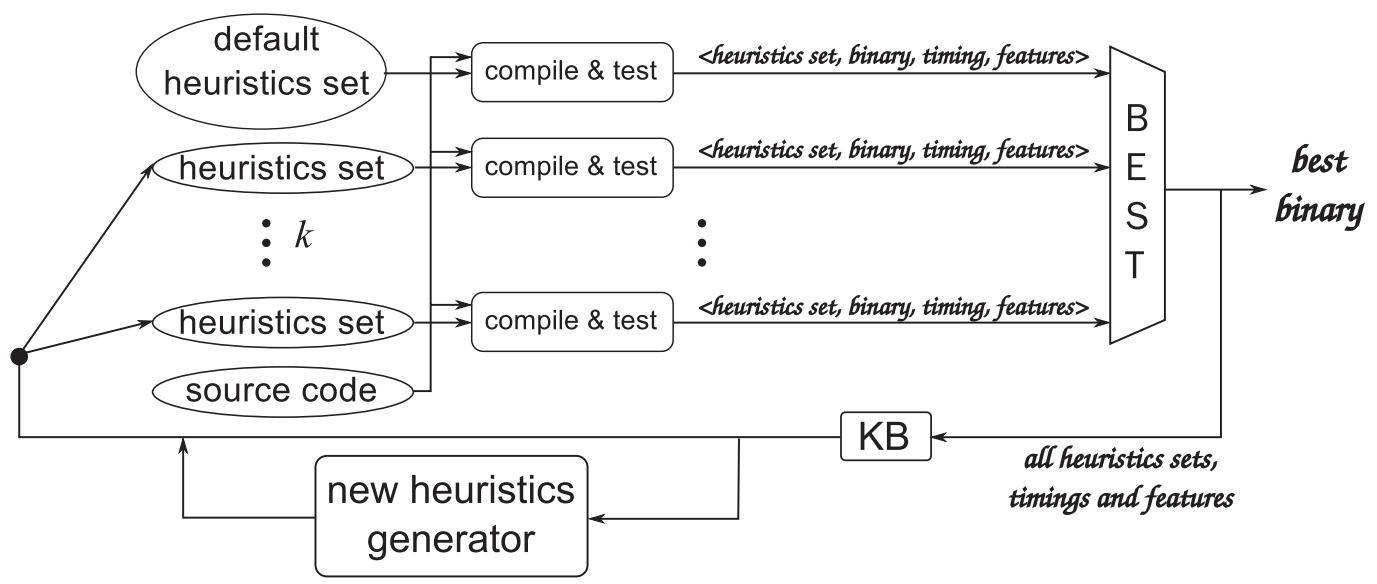

Fig. 1. The workflow of a compiler using long-term learning. The number of candidates to test is $k$.

candidates are derived from the old ones in a nondisruptive way. The idea is to start from a candidate configuration known to be good, and to modify it slightly, looking for a new configuration that yields better performance when applied.

First of all, the GETBESTCANDIDATES function of Algorithm 1 extracts from the knowledge base as many candidates as required to reach the user-specified number $k$ of candidates to test. They are taken from the fittest candidates (according to the fitness function described in Section 2.6) known up to the current time. Each heuristic is associated with a decision point name that univocally identifies in which phase of the compiler it has to be used. Every time the compiler needs to make a decision, it picks from its configuration the unique heuristic formula corresponding to that decision point. Therefore, each configuration must contain exactly one formula for each decision point name. The set of all possible names is denoted by $N$ in Algorithm 1 . Let $H_{i}$ be the set of the names of the heuristics contained in candidate $i$ extracted from the knowledge base. In case $H_{i}$ is not equal to $N$, the missing heuristics (identified by the names in $N \backslash H_{i}$ ) will be randomly generated by the GenerateMissingHeuristics function and used to complete the candidate just before testing it. This happens in particular when not enough candidates where found in the knowledge base: they are replaced by empty candidates, to be later filled in by GenERATEMissingHeuristics.

After the candidates have been extracted from the knowledge base, on each of them we execute the Evolve function with a given probability ( $p$ in Algorithm 1), meant to model the exploration versus exploitation trade-off [Kaelbling et al. 1996], that is the expected utility of trying new solutions instead of reusing the old ones, proven good. When the exploration decision is taken, Evolve determines the number of heuristics to evolve, the minimum being one and the maximum being a percentage $e$ of them. Then, the actual evolution process takes place for randomly chosen heuristics of the set. We allow more than one heuristic to evolve at once to enable a group of decisions that are effective only when applied together to be made at the same time.

When a formula is being evolved, it is parsed according to the grammar in Figure 2, obtaining a syntax tree where every leaf represents a numeric or boolean value or a feature name, and every internal node represents an operator or an if expression. Then, the tree is recursively visited, until a single terminal of the grammar is actually modified according to the following rules:

If expression $\left(\mathrm{IF}_{t}\right)$. Evolution is recursively applied to one element chosen randomly between the condition formula, the then case formula and the else case formula. 

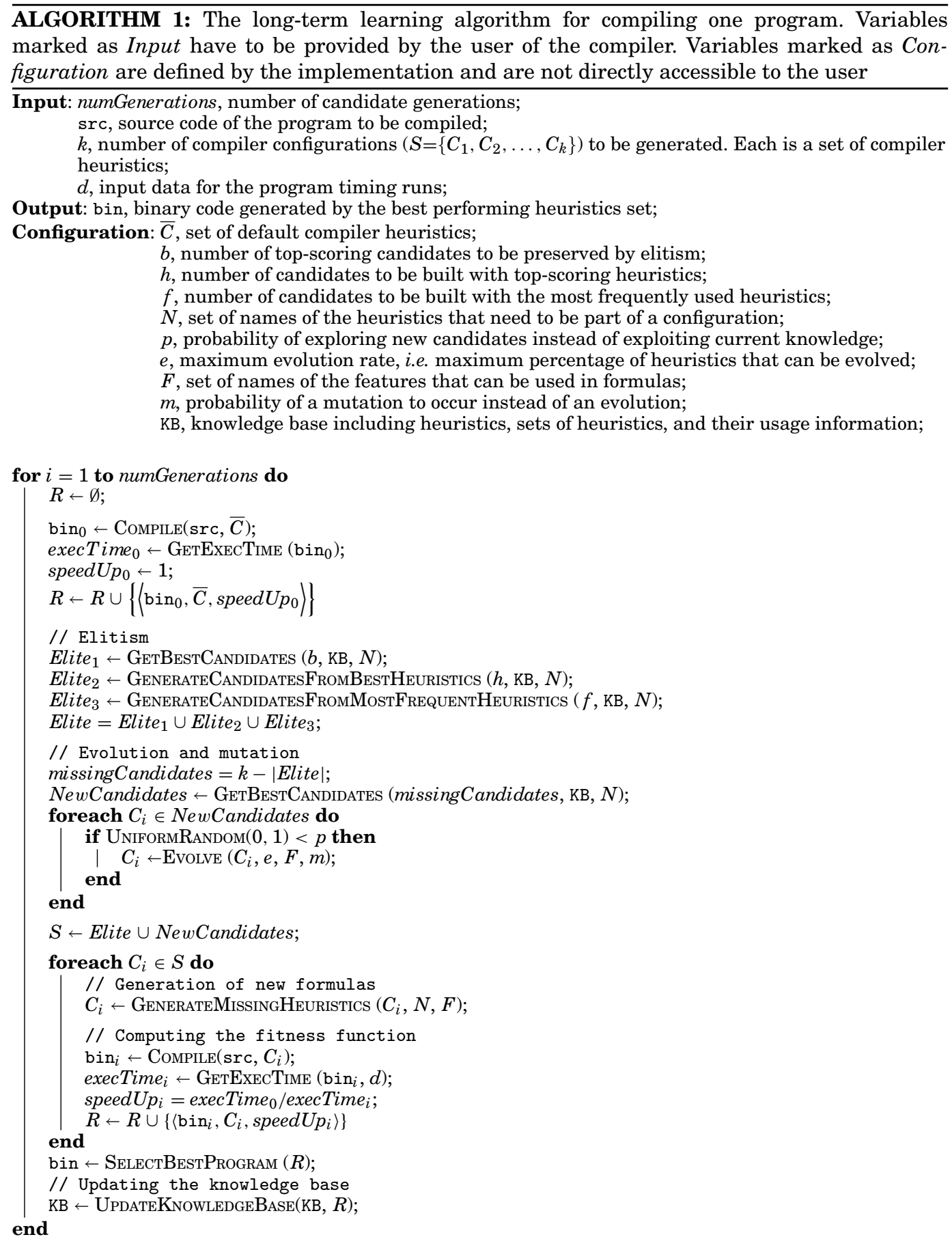

return bin, $K B$ 


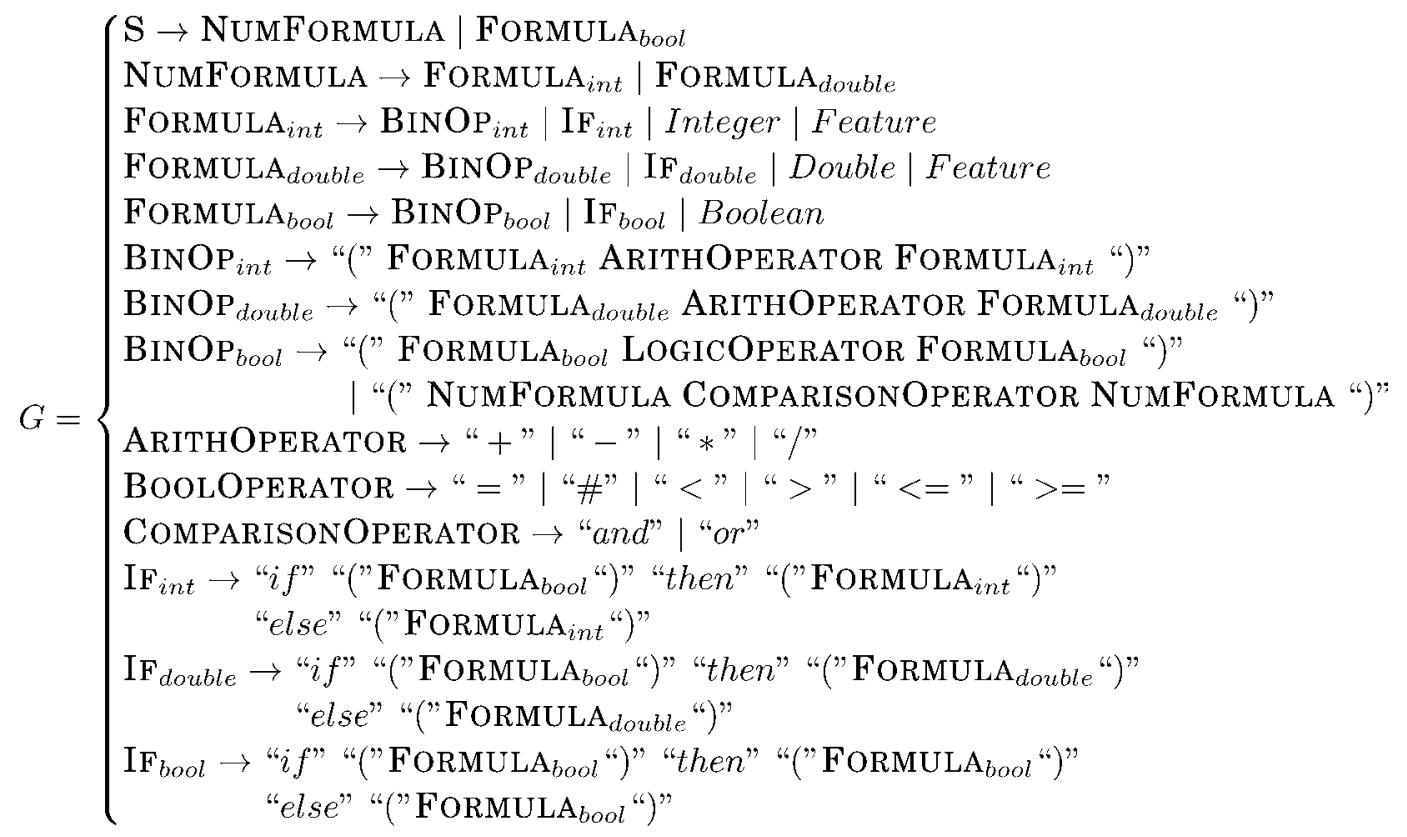

Fig. 2. Grammar representing all the formulas that can be used as heuristics. $\mathrm{S}$ is the axiom. Integer and Double represent, respectively, integer values and double-precision floating-point values. Features are represented in formulas as variables, each with a unique name. This will be substituted with their actual values by the compiler, when the heuristic is evaluated.

Binary operation $\left(\mathrm{BINOp}_{t}\right)$. Evolution is applied to one element chosen randomly between the left operand, the right operand, and the operator. In the first two cases, the evolution process continues recursively, in the last case the operator is substituted with a different one taken from the same category (ARITH, BooL, or COMP).

Boolean value (Boolean). The value is negated.

Feature (Feature). A different feature is used.

Numeric value (Integer or Double). The current value $v$ is incremented by a value in the interval $[-v, v]$. The resulting value can only be in the interval $[0,2 v]$ if $v>0$ or $[2 v, 0]$ if $v<0$. This is done to prevent the value from changing too much, under the assumption that a heuristic being evolved derives from one already evaluated as good, and therefore does not need to be drastically changed.

\subsection{Mutation}

Even if we modify multiple heuristics at once, there is a risk of getting stuck in local maxima, since evolution only applies small modifications to formulas. To avoid this, we implemented a mechanism able to provide bigger evolutionary steps, traditionally named mutation [Russell and Norvig 2010]: every time the evolution function is executed, it might be turned into a mutation with probability $m$.

The conceptual difference between evolution and mutation is in the editing distance. Evolution recursively visits the tree until it modifies a single terminal of the grammar. Mutation can be applied to either a terminal or a nonterminal and is able to change the formula much more deeply. The modifications applied to the formula are as follows:

Whole formula $(S)$. The formula is substituted by a completely new one.

If expression $\left(\mathrm{IF}_{t}\right)$. Either the condition, the then case formula or the else case formula is removed and substituted with a new subformula, generated from scratch. 
Binary operation $\left(\mathrm{BINOP}_{t}\right)$. Either the left or right operand subformula is substituted with a new subformula, generated using the grammar in Figure 2.

Boolean value (Boolean). Same behavior as evolution: the current value is negated.

Feature (Feature). Same behavior as evolution: a different feature is used.

Numeric value (Integer or Double). A random number is generated. It can be unbounded or, where specified, comprised in an interval determined by the validity range (see Paragraph "Validity range check" of Section 2.3) of the heuristic itself.

\subsection{Generation of New Formulas}

All the formulas that can be used as heuristics in our system can be described using the grammar in Figure 2. Every time a formula needs to be generated, the grammar is used as a generative device. The generation starts from axiom $\mathrm{S}$. The first choice (between Formula ${ }_{b o o l}$ and NumFormula) depends on the specific type of result that is needed. If the heuristic aims to decide whether or not to activate an optimization, a BoolFormula will be generated. If a compiler parameter has to be heuristically determined, NumFormula will be used, in one of its two versions (integer or double).

The choice of the right grammar is essential to generate good heuristics. We want to give the system enough flexibility to find good results. At the same time, as stated in Stephenson et al. [2003], the wider the heuristic search space, the longer it will take for the algorithm to converge upon a general solution. Any grammar could be expanded to contain more primitives able to produce more complex formulas, so it will never be possible to generate a completely unbiased set of heuristic formulas. Since we are defining an online learning system, it is particularly important for it to converge rapidly, so we choose to only use basic arithmetic and logic operands for the formulas. Machine learning techniques aim at being faster than iterative ones by biasing the search space in some way, and experimental results on multiple platforms and multiple test programs have proved our choice to be valid. Future work could look for more complex primitives able to deliver significant performance improvements.

2.3.1. Preventing Indefinite Growth of Heuristics. Formulas generated by the grammar are defined recursively and could be arbitrarily long. Should the grammar be used with uniform probability of choosing any of the alternative productions, the length of formulas would tend to increase rapidly, making them slower to evaluate and harder to read and understand. Generating readable heuristics is not required, but can be useful for compiler writers. When developing new optimizations, being able to easily understand how a heuristic makes decisions is handier than systems where the optimization decision process is less visible, such as the ones in Long [2011] and Fursin et al. [2011].

The importance of preventing a grammar from generating too long formulas is described in Leather et al. [2009] and Leather [2011]. They use a grammar to automatically define complex features to be extracted from programs and used in a classifier for implementing a machine-learning-based compilation algorithm. We use a similar approch, based on assigning weights to the various productions of the rules, but in our algorithm the set of features is fixed and we use the grammar to generate the heuristics the compiler will use to make decisions. In our specific case, weights are needed only for the rules describing formulas (i.e., Formula int , Formula double and Formula bool). The weights we use are described in Figure 3. The actual production to activate is chosen by a random roulette-wheel selection algorithm ${ }^{2}$.

For every other rule with more than one production $\left(\mathrm{BINOP}_{\text {bool }}\right.$, ARITHOPERATOR, BoolOPERATOR, COMPARISONOPERATOR) the selection is performed with a uniform probability, since all the alternatives generate formulas of equivalent length.

\footnotetext{
${ }^{2}$ Given a set of elements, each with a fitness value, a roulette-wheel selection algorithm chooses an element from the set with a probability proportional to the fitness of the element itself. We use weights as the fitness value.
} 


$$
\text { ForMula }_{t} \rightarrow \begin{array}{ll}
\operatorname{BINOP}_{t} & \text { weight }: 2 \\
\mathbf{I F}_{t} & \text { weight }: 1 \\
\text { FormulaType } & \text { weight }: 5 \\
\text { Feature } & \text { weight }: 4 \text { (only if } t \text { is int or double) }
\end{array}
$$

Fig. 3. The weights applied to the grammar to limit the expansion of heuristic formulas. FormulaType Fis respectively Integer, Double or Boolean for $t$ equal to int, double or bool.

When the Feature production is activated, the learning algorithm randomly chooses a feature from the set $F$ of the features whose value will be provided at compile time.

2.3.2. Reducing the Search Space Size. The search space of possible heuristics described by Figure 2 is infinite. The long-term learning evolutionary algorithm explores it efficiently, exploiting the acquired knowledge to avoid testing candidates likely to perform poorly, and focusing on evolving the sets of heuristics that have proven to be beneficial to the execution times of previously compiled programs.

Every restriction of the search space helps to speed up the convergence toward a useful solution. Therefore, we use two techniques to achieve this.

Constant folding. For every heuristic generated by the system, we apply constant folding techniques (e.g., if a fragment of a heuristic formula is $2 * 3+4$ or $1+2+3+4$ it will be folded to 10 in both cases) before storing the heuristic in the knowledge base.

This way, apparently different heuristics are reduced to a single one and the information gathered from those heuristics will converge into a single knowledge base record, making the data about that heuristic more complete and more useful. Having fewer heuristics makes evolution more efficient, since there are less starting points to use. Furthermore, this leads to simpler heuristics being stored.

Validity range check. The second technique we use is applicable only to non-boolean heuristics and is implemented as part of the scoring system (see Section 2.6). It has been introduced after observing that most numerical heuristics must generate results within a given range of values. For example, a heuristic determining the unrolling factor of a loop has 1 as the minimum acceptable value (meaning no unrolling) and could have a maximum around 100 (or it could also be left unbounded). A heuristic determining the number of worker threads a program should launch could be limited upwards by the number of cores actually available on the machine.

To take into account such boundaries, we introduced the possibility to specify a minumum value min and a maximum value max for every heuristic. The formulas we use are randomly generated, and they include as variables the features extracted by the compiler, therefore it is possible that their evaluation might result in a value outside the valid range. The trivial solution is substituting values below min with min itself and values above max with max itself. Such an approach ensures valid results, but impacts negatively on the heuristics: if the value producing the optimal executable corresponds to the min of the range, every formula evaluating to something below min would receive a high score. This is bad, because it would increase the number of formulas that are considered good, increasing the number of seemingly good starting points for the next generations. We decided instead to add a penalty (precisely described in Section 2.6) for formulas evaluated outside the valid range. Therefore, their score will become lower, favoring only the heuristics that stay within the boundaries.

In order to further reduce the possibility of generating low-scoring formulas, the min and $\max$ values are provided to the formula generation function, that exploits them to build formulas that cannot, by construction, be evaluated outside the validity range, as far as no variables (that is, features) are concerned. 


\subsection{Elitism}

If all the candidates in a generation are randomly generated, it might happen that their results are worse than the one obtainable by using the best set of heuristics known up to this point. Elitism is a common solution applied by evolutionary algorithms to this problem: it improves the stability of the learning process. Long-term learning uses a very reduced amount of candidates, so rapidly reaching stability is particularly important. Therefore, we implemented three different elitism mechanisms.

The GetBestCandidates function of Algorithm 1 (already described in Section 2.1) extracts from the knowledge base $b$ candidates whose heuristic formulas have been proved to be the highest-scoring ones when used as sets.

GenerateCandidatesFromBestHeuristics extracts from the knowledge base, for each needed heuristic name $n \in N$, the $h$ highest-scoring heuristics. Then, it combines them in sets, each containing one heuristic for each needed name. These sets are returned as candidates. Heuristics receiving high scores independently from the specific heuristic set they belong to are likely to receive a high score in a new set too.

GenerateCandidatesFromMostFrequentHeuristics extracts from the knowledge base, for each heuristic name $n \in N$, the $f$ most frequently used heuristics whose score is at least 1 (meaning they provide no slowdown, as described in the next section). Then it combines them in sets, each containing one heuristic for each name. These sets are returned as candidates. This is done because if an heuristics is frequently used without its score becoming too low, it means that, on average, the heuristic is able to provide acceptable performance. Reusing such an heuristic again provides a good fallback candidate, increasing the stability of the algorithm.

By analyzing the learning algorithm it is possible to determine why it could be slow to converge. Each of the elitism mechanisms aims at removing one cause of instability: the first by keeping the current best results across generations, the second one by forcing the creation of candidates likely to perform well, the third one by providing an acceptable fallback option in case every other candidate is not good enough. The third one is especially useful at the beginning of the learning process, where it might happen that the best set of heuristics performs well for all the programs compiled up to now, but still fails on a new program, too different from the previous ones.

\subsection{Knowledge Base}

The knowledge base stores information about all the tested candidates. In particular, for every heuristic, the knowledge base contains the name of the decision point of the compiler the heuristic will be used for, the formula of the heuristic, the score earned by the heuristic up to the current time, and the number of previous uses of the heuristics.

The knowledge base also stores all the sets each heuristics has been part of and, for each set, its score and its number of previous uses.

\subsection{Computing the Fitness Function and Updating the Knowledge Base}

For each candidate $i$, we record the execution time execTime ${ }_{i}$ and we compute the speed $U p=\frac{\text { execTime }_{0}}{\text { execTime }_{i}}$ with respect to the execution time execTime ef $_{0}$ of the default configuration $\bar{C}$. If speed $U p>1$, the candidate provides a performance improvement.

The UPDATEKNOWLEDGEBASE function uses the speedups as the fitness function of the heuristics and updates the scores contained in the knowledge base.

Every candidate contains a set of heuristics, each controlling a compiler decision. We keep track of how well the heuristics of the candidate work together by computing the score of the set of heuristics as a whole. At the same time, we keep a score for every single heuristic, to know how well it works whatever heuristic set it is part of. 
We also keep a use count for every heuristic set and for every heuristic. A set is counted as used every time it is used to compile a program. A single heuristic is counted as used every time it is evaluated, that is every time a decision is made based on its value. For example, a heuristic determining the unrolling factor of loops will be evaluated (and therefore used) every time an unrollable loop is being compiled.

The scores of both the heuristic sets and the heuristics are updated as

$$
\text { score }_{\text {updated }}=\frac{\text { score }_{\text {old }} * \text { usecount }_{\text {old }}+\text { score }_{\text {new }} * \text { validusecount }_{\text {new }}}{\text { usecount }_{\text {old }}+\text { usecount }_{\text {new }}},
$$

where score $_{\text {old }}$ and usecount $_{\text {old }}$ are the score and use count stored in the knowledge base, score $_{\text {new }}$ is the speedup measured during the test execution, usecount ${ }_{\text {new }}$ is the number of times the heuristic has been used during the test execution. If we are updating the score of a heuristic set, usecount $_{\text {new }}$ will always be equal to one. validusecount ${ }_{\text {new }}$ is the number of times the result of the evaluation of the heuristic was inside the validity range

$$
\text { validusecount }_{\text {new }}=\text { usecount }_{\text {new }}-(\text { tooLow }+ \text { tooHigh })
$$

with tooLow (respectively tooHigh) being the number of times the heuristic was evaluated lower (respectively higher) than min (respectively $\max$ ).

Immediately later, the use count is incremented too.

$$
\text { usecount }_{\text {updated }}=\text { usecount }_{\text {old }}+\text { usecount }_{\text {new }}
$$

On the other hand, it might happen that a candidate configuration fails to generate a working executable, usually because some optimizations of the underlying compiler cannot be applied at the same time. If a configuration failed, we want to penalize it. Therefore we use a score $_{\text {new }}$ equal to zero and a usecount $t_{\text {new }}$ equal to 1 , thus decreasing its score, especially if this happens when the heuristic that led the configuration to fail is fairly new and its usecount

As it can be seen from Equation (1), the score that is computed along the life of the knowledge base is the expected speedup, of each heuristic set and of each heuristic. It is used by the algorithm to determine the best known candidates: the higher the score, the better the expected speedup while using the heuristic.

Rationale. The current scoring system was chosen because it proved experimentally ${ }^{3}$ to be fast at learning when no knowledge is available, while keeping the ability to improve later on. Furthermore, the method we use to update the score of each heuristic scales well with the number of their executions (the cost of update is constant). This is extremely important, since we collect data for every single candidate we test.

It is worth noting that we considered multiple scoring algorithms for long-term learning. At first, we considered an algorithm that sorted the candidates by speedup, then assigned a score based on the ranking (for $n$ candidates, decreasing from $n$ points for the best one to 1 for the worst one). Such an algorithm is more robust to measurement disturbance during the test phase, since the score is unchanged as long as there is no switch in the relative positions of one candidate with respect to another one, whereas using the speedup is sensitive to such errors. We decided to use the speedup itself, instead of the speedup ranking, to determine the score for two reasons. First, it provides useful information discerning how better a candidate is compared to another one (e.g.,

\footnotetext{
${ }^{3}$ This experimental choice does not reduce the generality of our approach, because only a reduced number of programs (namely bitcount, qsort1 and susan_c) were used during the development of the GCC version to make this decision. Later, performance results over the full set of benchmarks and the implementation in a second compiler just confirmed the quality of the chosen scoring system.
} 
being twice as fast is not as good as being four times as fast). Second, it takes into account whether a candidate provides an actual improvement: using the ranking-based system, if all the candidates in a set are slower than the default configuration, the best one would still get the maximum number of points, making it an apparently good candidate. This problem could be partially solved by assigning zero points to candidates with speedup below 1 , but this would still lose interesting information: a candidate scoring just below 1 might be a good one when applied to a different program, and penalizing it too is likely a short-sighted decision.

\subsection{Compiler Performance over Time}

The long-term learning algorithm does not have an initial training phase: it can immediately be used. Therefore it is important to determine what is the performance of a pristine system based upon such an algorithm.

A strict requirement of the algorithm is the availability of a default configuration, to be used as the comparison term to compute the speedup of each candidate. It can be a configuration where all the optimizations are disabled and all the numeric parameters of optimization algorithms use generic values: the algorithm does not require any pre-existing knowledge about the underlying hardware architecture and the expected benefits of the compiler optimizations. It is its task to figure this out. Nevertheless, if there is already available knowledge, the algorithm is able to exploit it. The best way to do this is to use such knowledge to define a nontrivial default configuration.

Since the default configuration is used as the comparison term, only the candidates improving upon it will receive a good score: the default configuration can be seen as the worst-case fallback the system uses when it cannot find a better configuration.

The ideal usage for long-term learning would be to enhance the performance portability enabled by iterative compilation algorithms [Dubach et al. 2009]: the compiler could be distributed with a generic configuration, containing a set of heuristics good for a family of architectures (e.g., x86-i386 architectures). While a program is being developed, every time it is compiled, the compiler evolves as well, adapting itself to the specific system it runs on. When it is time to release the production binary, the evolution of heuristics can be disabled if the need to keep the performance predictable arises. In order to further speedup the evolution of heuristics, the knowledge base can be shared by all the developers working on a project. This would also prevent them from dealing with different binaries.

Long-term learning is meant to constantly improve the performance of the compiler over its whole life. It would be unrealistic to think that the target system is never going to change and that the compiler itself is never going to be updated. Therefore, the algorithm was designed from the beginning with the objective of being robust to this kind of events.

The target system changes. First of all, let us consider the case of the unmodified compiler with a target system that changes (a new processor is installed, the amount of available RAM is increased, a new version of the operating system is installed, etc.). If the current best heuristics sets performs well with the new system configuration, nothing will change. On the other hand, if some new heuristic obtains better performance, it will be chosen more often for originating new candidates. At the same time, the score of the old heuristics will slowly decrease, until they drift into oblivion.

Having a new heuristic reach the top of the ranking might seem hard, but it is not. New heuristics are able to overcome the old ones because all the scores are determined by the speedups weighted by the number of times every heuristic has been used. Therefore, old heuristics are resilient to changing their score (since they have proven 
their average speedup over time) but new ones have low weight and their score can immediately obtain a really high value if they provide a high speedup.

Changing program features' availability. Let us consider now the availability of a new version of the compiler, able to compute a new program feature. All the old heuristic formulas are still valid. During the evolution and mutation processes, new formulas are generated, and some of them will use the new feature. If they manage to get higher speedups, they will earn higher scores and will be used more frequently.

In the opposite scenario, should the compiler lose the ability to compute a feature, the heuristic formulas containing that feature will not be able to be evaluated any more. This means their score will drop fast, and they will stop being used.

New heuristic needed. Let us consider the scenario where an updated version of the compiler has to take a new decision and therefore needs a completely new heuristic. Every candidate configuration used in the past is composed by a set of heuristics (one for each decision point in the compiler). The names of all the needed heuristics are stored in set $N$ of Algorithm 1. When the compiler requires a new heuristic, its name is added to $N$. The GenerateMissingHeuristics function is called immediately before using each candidate. If a name in set $N$ has not a corresponding heuristic inside the candidate, GenerateMissingHeuristics will generate the formula for such heuristic, completing the candidate.

One compiler, different histories. With long-term learning, every deployed compiler has its own compilation history and has generated different heuristics, thus behaving differently from every other compiler. This might have downsides, especially in a production environment, when it comes to bug reporting. On the other hand, it should be considered that this is no different from every other machine-learning-based compilation approach: after the training phase, each of these compilers has its own, unique model, and modifies its own behavior accordingly. We designed the knowledge base to be as small as possible and contained in a single file. This allows different developers to easily exchange their history, when needed, such as for bug fixing or for comparing performances. Furthermore, the learning process can easily be disabled, when needed, using only the current best heuristics. This allows to have a traditional compiler, but with ad hoc heuristics, once the desired performance level has been achieved. As for other machine learning approaches, deciding when to stop the learning process is up to the user: the longer, the better. But unique to long-term learning is the possibility to never stop it: idle CPU times could be used to compile more programs, therefore further improving the knowledge and the performance.

\section{EXPERIMENTAL SETUP}

In order to show the viability of our approach and its applicability to multiple compilers, we implemented long-term learning into two different compilation toolchains (GCC and PetaBricks), and performed tests on multiple hardware configurations.

The long-term learning algorithm implementation is divided into two parts. The learning algorithm itself is implemented in Python as a framework, and is common to all the compilers. It chooses and learns the heuristics. The second part is implementation specific and it gives the compiler a way to provide the program features to the algorithm and to use the chosen heuristics to make decisions about the compilation process. The compiler-specific implementations will be described in Section 3.1 and 3.2. In these experiments, most of the times the heuristics are then used to decide the best command line parameters, but this is just one possible use: in fact, the heuristic for the Petabricks tiling algorithm is evaluated directly inside the compiler itself. 
Table I. The Values We Used for the Parameters of Algorithm 1, Obtained by Experimentation

\begin{tabular}{ll}
\hline Parameter & Value \\
\hline top-scoring candidates preserved by elitism $(b)$ & 1 \\
candidates built with top-scoring heuristics $(h)$ & 1 \\
candidates built with most frequently used heuristics $(f)$ & 1 \\
needed heuristics $(N)$ & compiler-dependent: \\
& see Section 3.1 and 3.2 \\
exploration probability $(p)$ & 0.2 \\
maximum evolution rate $(e)$ & 0.3 \\
available program features $(F)$ & see Figure 4 \\
mutation probability $(m)$ & 0.3 \\
\hline
\end{tabular}

As it can be seen in the Configuration section of Algorithm 1, long-term learning needs a few parameters to be set in order to define its behavior. We determined the values to assign to such parameters (in Table I) through a brief experimentation.

Our implementations were tested on different hardware configurations, to show that the algorithm is able to adapt compilers to multiple architectures.

Hardware Configuration 1. A machine with 4 Intel Xeon X7550 processors (8 physical cores each) running at $2.00 \mathrm{GHz}$ with $128 \mathrm{~GB}$ of RAM.

Hardware Configuration 2. One core of a machine part of the CILEA Lagrange cluster [Arlandini and Invernizzi 2008]. Each machine is equipped with two Intel Xeon X5460 processors (4 physical core each) running at $3.16 \mathrm{GHz}$ and 16GB of RAM.

Hardware Configuration 3. One processor of a machine equipped with two Intel Xeon X5460 processors (4 physical cores each) running at $3.16 \mathrm{GHz}$ and 8GB of RAM.

We want to show that the knowledge acquired by the compiler through long-term learning can be applied to improve the performance of new programs. Therefore, we need to simulate the life of a compiler that has compiled a certain number of programs previously, showing how this affects the performance of an unseen program.

We have a set of available programs, taken from a benchmark suite. For every simulation, we use one of the programs of the suite as the test program and the rest as the training set, in a Leave-One-Out Cross-Validation [Kohavi 1995] configuration.

Each simulation is performed as follows. First of all, the test program is compiled using the default compiler configuration to find out the reference speed. Then, a program from the training set is compiled. Then, the test program is compiled again, using the knowledge acquired by the compilation of the first training program, and is then tested recording its execution time. Later, we compile the second program from the training set and then again we compile and run the test program, using the knowledge acquired from the compilation of the first two training programs. This procedure is repeated for each program in the training set.

It should be noted that, in order for the experiment to be meaningful, it is mandatory that the test program has never been seen by the system before, to prevent the compiler from acquiring knowledge from its compilation. In the simulation setup that was just described, the test program is compiled multiple times. In order to allow this, a special option was implemented in the compilers, enabling a program to be compiled using the current knowledge, but disabling write access to the knowledge base. Such option is used every time the test program is compiled, therefore, every time, the system behaves as if it never saw such program before.

It is also worth noting that we use the term "training set", but this does not mean that a training phase is needed: it just denotes the set of previously compiled programs, that provide the data for the learning process. In a production system, such programs would be the programs compiled by the compiler during its lifetime, up to the current one. The compiler is immediately usable, without an explicit initial training phase. 


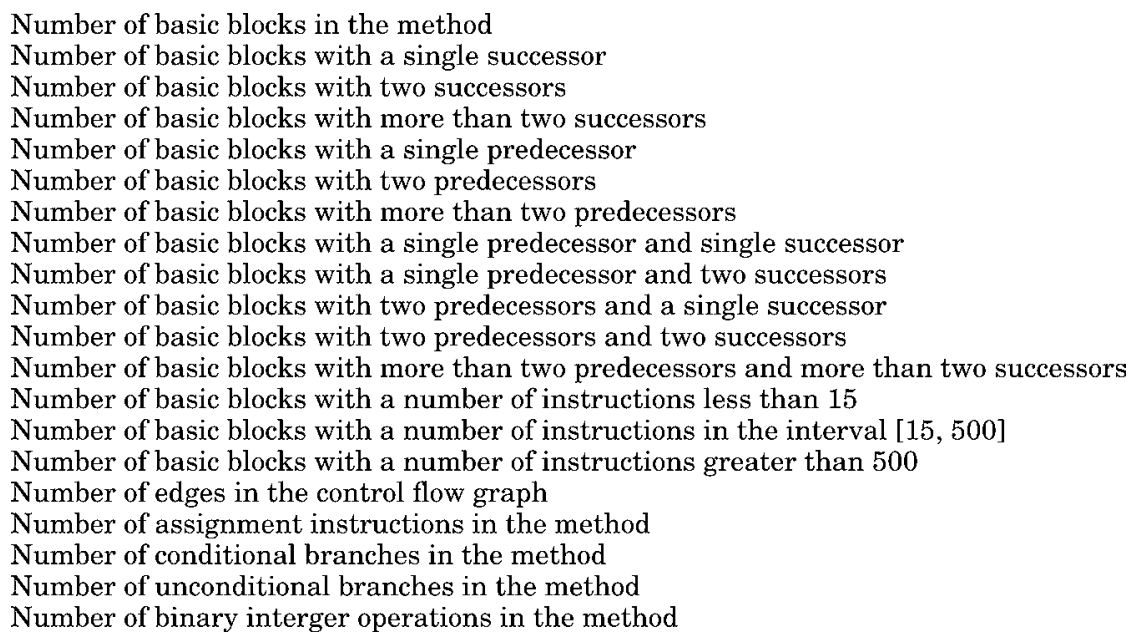

Fig. 4. The list of features used in heuristic formulas.

Since the long-term learning algorithm performs many operations randomly, it is expected that two different simulations will yield different results. Therefore, in order to provide more statistically relevant information, all the graphs shown in Section 4 are plotted using data computed as the average execution time computed over 5 runs.

The next two sections will describe compiler-specific details of our implementations.

\subsection{GCC}

We implemented long-term learning on top of GCC 4.6.3. The compiler-specific part consists of two components. The first component is a GCC plugin, activated during the compilation process to extract the static features (that is, extracted from code analysis, as opposed to dynamic features, extracted from runtime information) of the program. The computed values refer to the GIMPLE [Merrill 2003] intermediate representation of GCC. We decided to use a subset of the features proved valid by Namolaru et al. [2010], namely those listed in Figure 4, each averaged on the values computed for the whole program being compiled. The features we chose are simple but able to describe the structure of the program: they allow us to automatically find out heuristics at least as good as the hand-written ones provided by GCC. We might implement more feature extractors in the future, to better characterize the programs and further optimize them. The second component is the interface between the Python learning framework and GCC itself. It is responsible for evaluating the heuristics and invoking GCC enabling the optimizations through GCC command line flags according to the evaluated heuristics. Unfortunately, GCC doesn't allow full control over the specific optimizations to be enabled, since many of them depend on a specific optimization level being activated. Therefore, instead of just learning the list of optimization passes to enable, long-term learning actually learns both the optimization level (between -00 and -03$)$ and the list of optimization flags to enable (-f<optimization_name>) or disable (-fno-<optimization_name>) explicitly. Is is known that some of GCC's optimizations are disabled by default because they are unsafe. We deal with this by automatically discarding executables that turn out to be not working after being generated.

The benchmark suite we used for GCC is cBench [Fursin 2010]. It is composed by sequential C programs (listed in Figure 5) and derives from MiBench [Guthaus et al. 2001], with modifications aimed at making it better to test the efficiency of compiler optimizations. We chose this benchmark suite instead of other well-known ones (such 


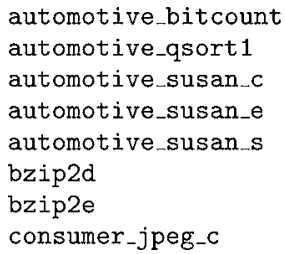

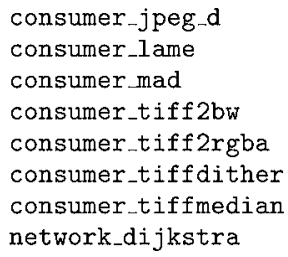

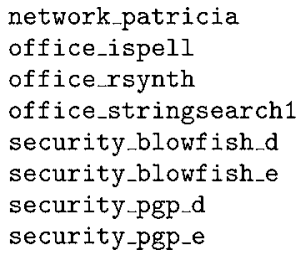

security_rijndael_d security_rijndael_e security_sha telecom_adpcm_c telecom_adpcm_d telecom_CRC32

telecom_gsm

Fig. 5. The list of programs used for the experimental tests.

as SPEC) because it is used by many other works dealing with machine learning and iterative compilation, making it easier to compare the results. In cBench, each benchmark is associated with a number representing how many executions (usually hundreds) of the program are required to obtain a total running time of about 20 seconds for the unoptimized version. We use such total running time as the value we compute the speedup on, because the multiple executions smooth out measurement errors. cBench contains multiple datasets for each program. Nevertheless, we just used one dataset for each program, because Fursin et al. [2007] showed it sufficient to achieve an optimization level within $5 \%$ of the best possible level. We might consider, at a later stage, to modify the implementation to use different datasets for each generation of candidates. cBench programs are sequential, so they are not influenced by the fact that we are using parallel hardware to run them.

\subsection{PetaBricks}

PetaBricks [Ansel et al. 2009] is an open-source compiler and programming language developed at MIT that uses machine learning and evolutionary algorithms to autotune [Ansel et al. 2011] programs, by making both fine-grained and algorithmic choices. PetaBricks programs work on numerical matrices as their input and output data. The compiler produces executables that expose hooks allowing the autotuner to adapt them to the underlying platform and to a given input data size.

PetaBricks does not compile $\mathrm{C}$ programs, therefore, we used its own benchmark suite instead of cBench to perform our tests.

The PetaBricks compiler is a source-to-source translator, reading its own input format and generating $\mathrm{C}++$ programs that will be later compiled by GCC, used as the backend compiler. Therefore, we reuse part of the setup from the GCC long-term learning compiler. In particular, we use the same GCC plugin to compute features about the program being compiled. The plugin works at GIMPLE level, so the source language ( $\mathrm{C}$ for the cBench tests, $\mathrm{C}++$ for PetaBricks-generated code) is not important. At first, we considered using features collected from PetaBricks's own intermediate representation (IR), but PetaBricks has not many optimizers requiring heuristic decisions, so we mainly use them to direct the optimizers of the underlying GCC. Therefore, such IR is too high level to be useful for our actual setup. Though, given more PetaBricks optimizers, PetaBricks' own IR would provide more interesting features.

The interface between the long-term learning framework and PetaBricks is written in Python, as for GCC, but instead of evaluating the heuristics it just stores them in a configuration file passed to PetaBricks as a parameter. We modified the PetaBricks compiler adding a component able to evaluate the heuristics. This shows that it is possible to use long-term learning with different levels of integration with the compiler.

The optimizations the PetaBricks implementation works on include a PetaBricksspecific parameter, influencing the code it generates: the number of times matrices have to be split by the tiling optimizer of PetaBricks. Furthermore, we learn the numerical optimization parameters that are passed to the underlying GCC compiler, namely those related to function inlining (max-inline-insn-auto, 
max-inline-insns-single, large-function-insns, large-function-growth, large-unit-insns, inline-unit-growth) and to loop unrolling (max-unroll-times, max-unrolled-insns), because PetaBricks generates $\mathrm{C}++$ code containing many small functions and many loops. We also learn all the flags described in Section 3.1.

We learn more optimization parameters than in the GCC implementation: this is not related to the speed of the learning process or the speedups we obtain with or without them. It is only meant to show the flexibility of our algorithm. In the GCC implementation we learn heuristics to make binary decisions about optimization flags. Here, we also show the possibility to learn heuristics that evaluate to numerical results, both for direct use with PetaBricks optimizations or with optimization of underlying GCC.

We choose not to integrate the long-term learning algorithm and the autotuner because autotuning aims not only at improving the performance of the program, but also at making it able to adapt to changing hardware without the need to recompile the software [Ansel et al. 2009]. Long-term learning only modifies the program at compile time, though long-term learning could be used to automatically determine good default values for all autotunable parameters, enabling immediate execution without the need to autotune the programs.

\section{EXPERIMENTAL RESULTS}

In this section we will present the results of the tests we performed using the described experimental setup, first for GCC, then for PetaBricks. Unfortunately, it is not possible to provide a direct comparison with other learning-based systems, since the main aim of long-term learning is to be able to find out heuristics for compilers that have none, while other systems aim to improve the performance of the existing heuristics.

\subsection{GCC}

We performed multiple tests using GCC. We used both -00 and -03 as the default configurations. The first configuration allows us to verify the ability of long-term learning to automatically find out good heuristics for a compiler with not even a default set of heuristics defined yet (here, we aim at reaching the same performance as -03 from -00 ). The second configuration enables most of GCC optimizations, and is used as a reference by most iterative compilation works. When using it as the default configuration, we aim at learning heuristics able to improve over its performance.

Figure 6 shows the results of long-term learning on Hardware Configuration 1, using automotive_susan_c as the test program. The initial, unoptimized, execution time drops rapidly as soon as knowledge from the compilation of at least one other program has been acquired, because the system manages to find out a set of heuristics enabling the optimizations that deliver the most speedup. Later, compiling more programs, the execution time continues to decrease slowly, as marginally better heuristics are found.

The number of different configurations that are tested during one compilation with long-term learning is determined by parameter $k$ of Algorithm 1 . Figure 7 shows that, on Hardware Configuration 1, using just 5 candidates per generation leads to a slowly declining learning curve, whereas using more than 6 candidates per generation does not provide significantly faster learning, so we chose to set the default to 6 . We also determined experimentally that our algorithm performs well with just 3 generations. Therefore, with less than 20 candidate configurations tested during each compilation we can obtain good results without the need for an initial training phase, whereas other iterative approaches need hundreds of them. It should be noted that using 6 candidates over 3 generations leads to better results than compiling 18 candidates in a single generation because when using a single generation all the candidates are randomly generated starting from the pre-existing knowledge of the compiler, whereas using 


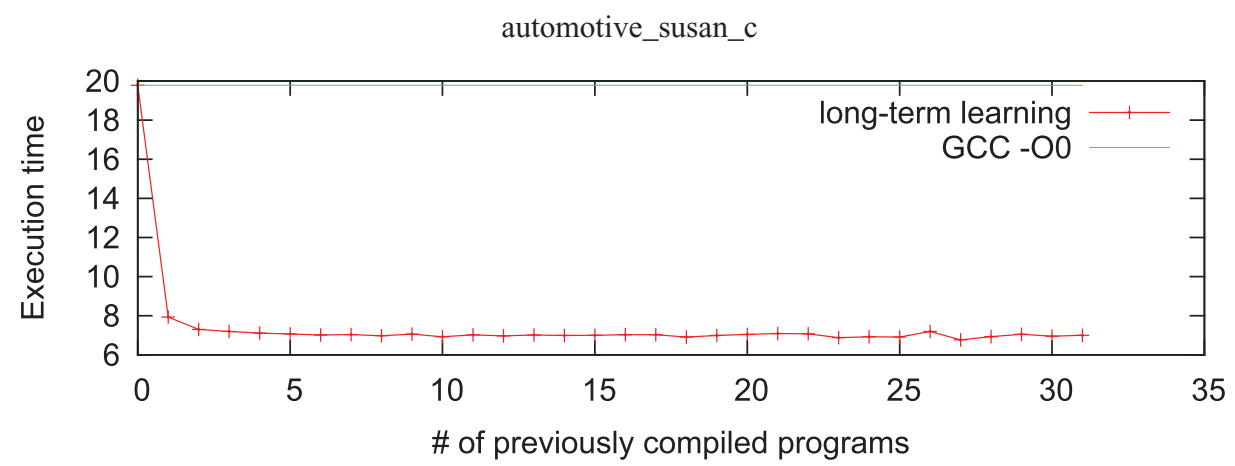

Fig. 6. Execution times of automotive_susan_c on Hardware Configuration 1 after the compilation of an increasing number of other unrelated programs. The default configuration is -00 .

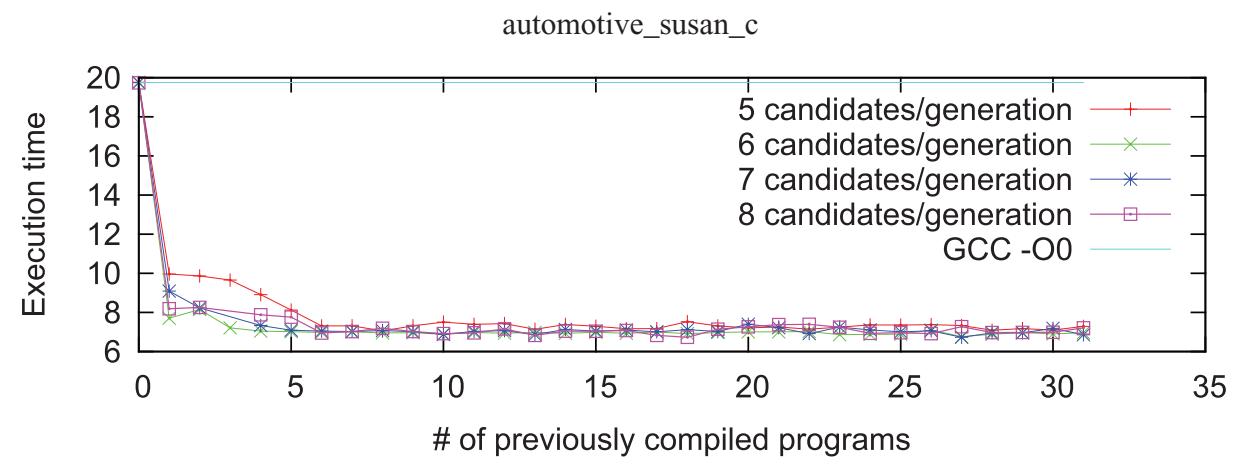

Fig. 7. Execution times of automotive_susan_c on Hardware Configuration 1 for varying number of candidates used for each generation. The default configuration is -00 .

multiple generations the candidates of each generation are based upon the knowledge acquired during the previous one for the specific program being compiled.

Figure 8 shows the execution times for the same test on Hardware Configuration 2, using different training sets. Training set 1 is the same used for Figure 6 . The training sets 2 and 3 include the same programs of training set 1, but in a different order. As can be seen, the behavior of the different training sets is comparable. The descending trend is analogous for the three sets. Training set 2 is included in the graph to show that even if the initial choice of heuristics is not optimal, the performance tends to improve and to converge to the minimum over time. Therefore, except for the very first programs, the specific sequence of previously compiled programs is not very important in determining how well the next programs will be compiled.

Figure 8 also shows us that long-term learning is able to devise heuristics that reach and surpass the performance of -03. Unfortunately, though, this does not always happen: Figure 9 shows an example of a test program where on average, long-term learning is able to optimize with respect to the provided default configuration, but without reaching the performance of -03. Nevertheless, given enough previously compiled programs, or a specific execution where the long-term learning algorithm explores the heuristics' search space in a particularly convenient way, it will eventually converge to the best possible solution, improving over -03 where possible. If the aim is to actually improve the performance of -03 , the best approach is to set such level as the default configuration to compute the speedup against. This way, as described in Section 2.6, all the 


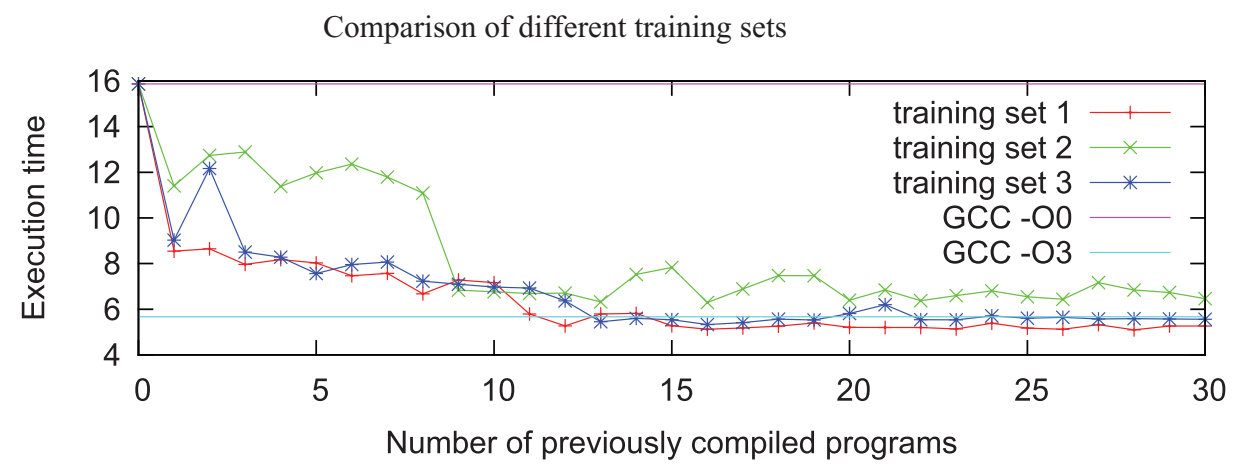

Fig. 8. Execution times of automotive_susan_c on Hardware Configuration 2 after the compilation of an increasing number of other unrelated programs. The test is repeated using different training sets. Execution times improve both on gcc -00 and on gcc -03 for training sets 1 and 3 . The default configuration is -00 .

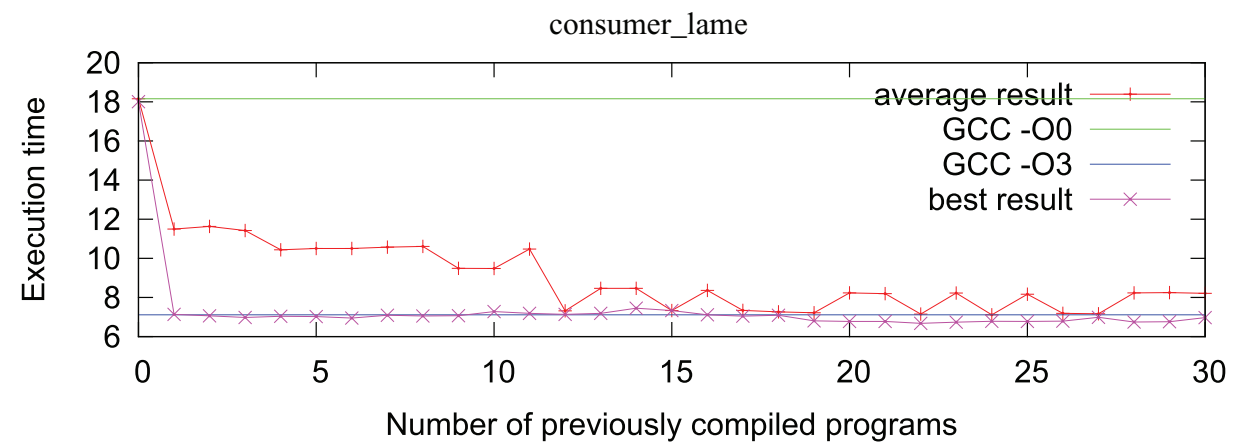

Fig. 9. Execution times of consumer_lame on Hardware Configuration 2 after the compilation of an increasing number of other unrelated programs. The average execution times improve on gcc -00 but not on gcc -03 , though they can be surpassed if the random generation of heuristics explores the right area of the candidate heuristics' search space.

configurations yielding an execution time higher than the default configuration will be penalized: the algorithm selects heuristics actually improving over the default one, if they exist. Figure 10 provides an example of such an improvement. It shows how long-term learning is able to improve the performance of the telecom_gsm benchmark over GCC -03. It is worth noting that the improvement is not as stable as those over -00. This is due to the fact that the execution times of good configurations are close to those of bad configurations. Therefore, the score assigned to good heuristics sets is only slightly higher than that of bad sets, making the choice of the best ones more tricky.

It is interesting to inspect the behavior of long-term learning over a longer time span (90 programs in the training set instead of 30), shown in Figure 11. Here we can clearly identify three different phases. At the beginning (until 10 programs have been compiled) the learning process is really fast, since it is easy to learn optimizations that provide a huge speedup. Actually, some overfitting takes place: the first 10 programs of this test contain some similarities with automotive_susan_c, therefore its performance improves very fast. Nevertheless, the aim of long-term learning is to optimize the compiler to perform better on average, for all programs. The test program is not treated in a special way. Therefore, as the long-term learning-enabled GCC goes on compiling new programs from the training set (programs between 10 and 50), the performance of automotive_susan_c gets worse, but is unstable. After the first 50 training programs 


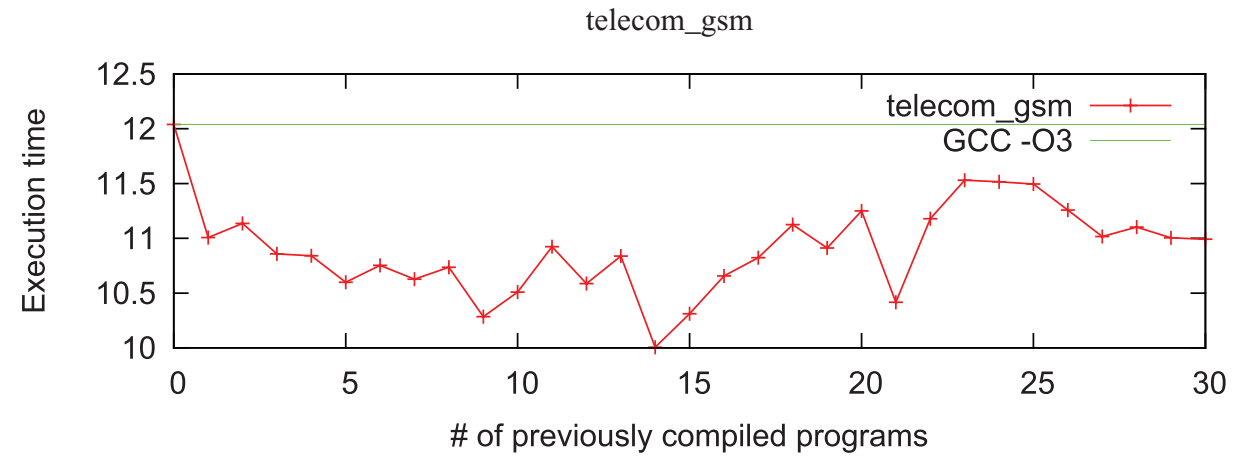

Fig. 10. Execution times of telecom_gsm on Hardware Configuration 1 after the compilation of an increasing number of other unrelated programs.

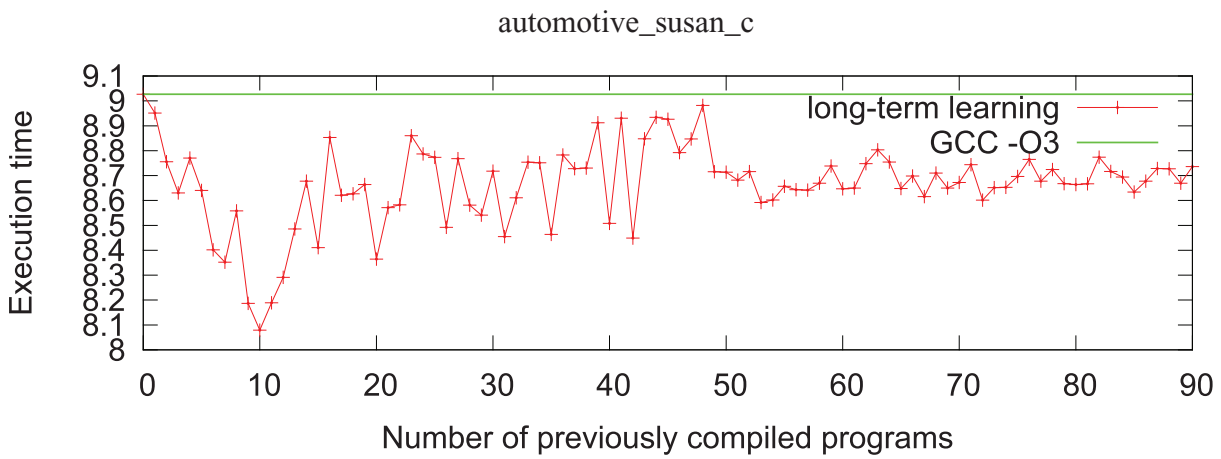

Fig. 11. Execution times of automotive_susan_c on Hardware Configuration 1 after compiling an increasing number of other unrelated programs. The performance level improves while becoming more stable over time.

have been compiled, enough knowledge has been gathered for the performance to become more stable and predictable. It is likely that, given even more training programs, after finding out an heuristic set that is good, on average, for all programs, the system would tend to converge to a heuristic set that is able to predict the best configuration for every program, therefore further improving the performance again.

Figure 12 shows the average and maximum speedup obtained by each of the test programs along the lifetime of the compiler over the default configuration -00 on Hardware Configuration 2, and compares them with the speedup over GCC -03. More precisely, let $s_{i}$ be the speedup of the test program after compiling $i$ training programs, and let $n$ be the total number of training programs. The values plotted in the bar chart are computed as

$$
\text { average speedup }=\frac{\sum_{1}^{n} s_{i}}{n} \text { and maximum speedup }=\max \left(\left\{s_{1}, \ldots, s_{n}\right\}\right) .
$$

The average speedup includes the small speedups obtained after compiling the first few programs, so, obviously, it is worse than GCC -03. But in the end, as by our aim, the algorithm is able to find heuristics good enough to reach and surpass -03, as shown by the maximum speedup.

Figure 13 shows the speedups obtained over GCC -03. As can be seen, the speedups obtained over -03 are not too big, but still comparable to those of GCC profile-driven optimization. This was expected. The main aim of the long-term learning algorithm is 


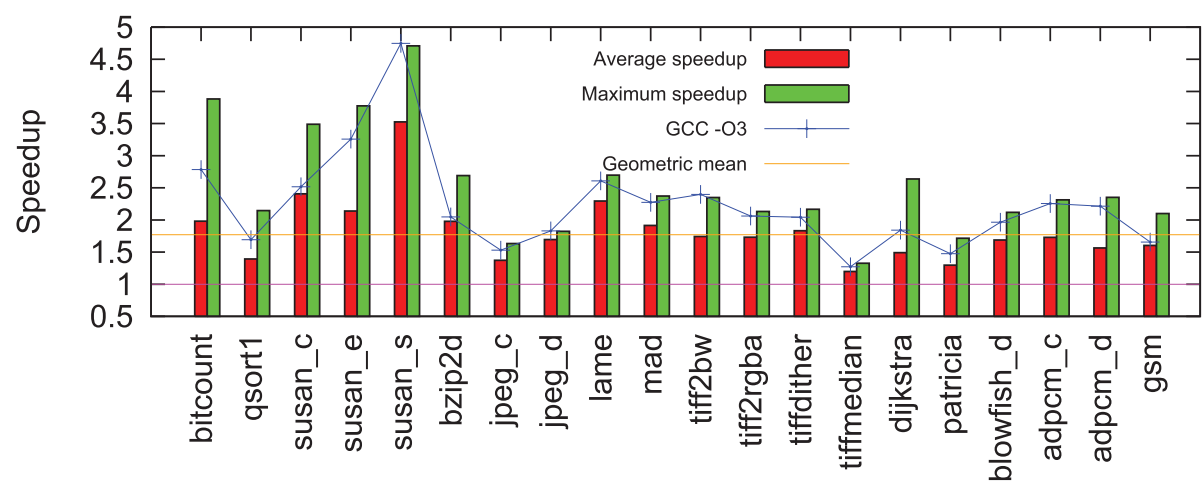

Program name

Fig. 12. Speedup obtained by various programs with respect to gcc -00 on Hardware Configuration 2, and compared to gcc -03 . The average and maximum speedup are computed over all the values obtained by testing the program once after compiling each of the programs listed in Figure 5.

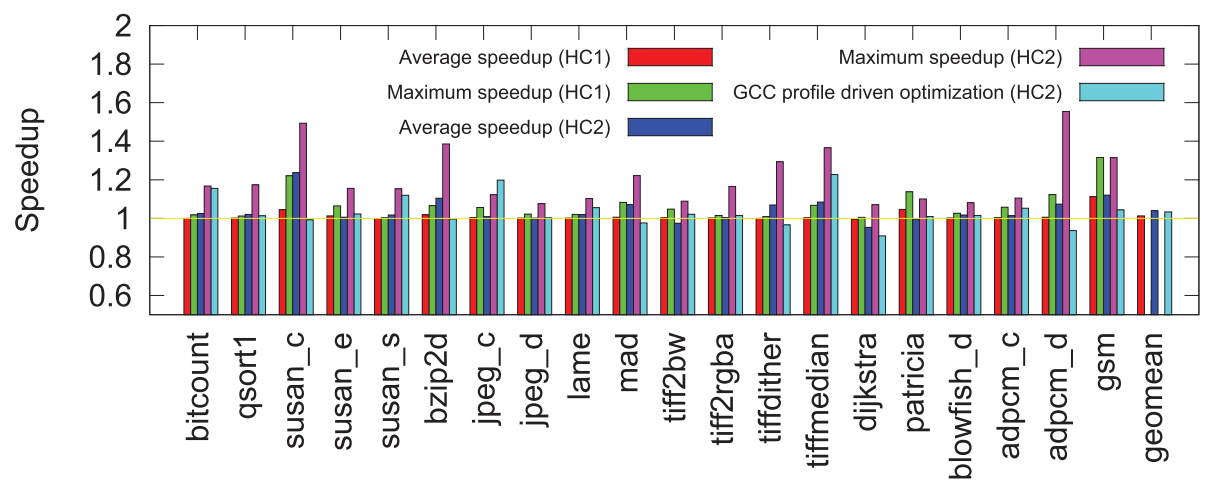

Program name

Fig. 13. Speedup obtained by various programs with respect to gcc -03 on Hardware Configuration 1 (HC1) and 2 (HC2). We represent the average and maximum speedup computed over all the values obtained by testing the program once after compiling with long-term learning each of the programs listed in Figure 5 . The speedups provided by GCC profile-driven optimization are presented as a comparison. The last bar, geomean is the geometric mean of the measured speedups, computed for HC1, HC2 and HC2 with profile-driven optimization.

to find a model of the target architecture, in the form of a good compilation heuristics set, without the need for human intervention in the process. The data we gathered show that the algorithm is able to reach the performance of GCC's maximum optimization level without the need for any training phase. Ongoing work is trying to always ensure a sensible improvement over -03.

\subsection{PetaBricks}

The amount of data we gathered about PetaBricks is smaller than that available for GCC, because the running time of the tests is longer, for various reasons. First of all, the number of tests contained in PetaBricks's benchmark suite is bigger than that of cBench. Furthermore, every program compiled by PetaBricks needs to be tuned by the underlying autotuner that adapts runtime parameters exposed by each program to the system it is running on, using the algorithm described in Ansel et al. [2011]. It is a genetic algorithm, and as such it requires the program to be run multiple times 


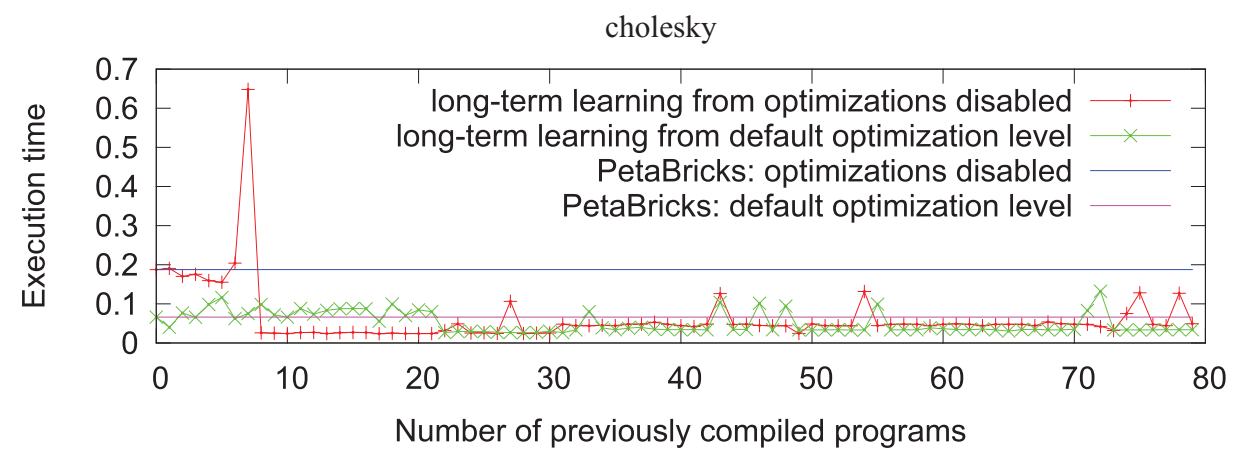

Fig. 14. Execution times of cholesky on Hardware Configuration 1 after the compilation of an increasing number of other programs. The speedup is computed with respect to PetaBricks with no optimization knowledge at all (optimizations disabled) and from the default optimization level.

cholesky

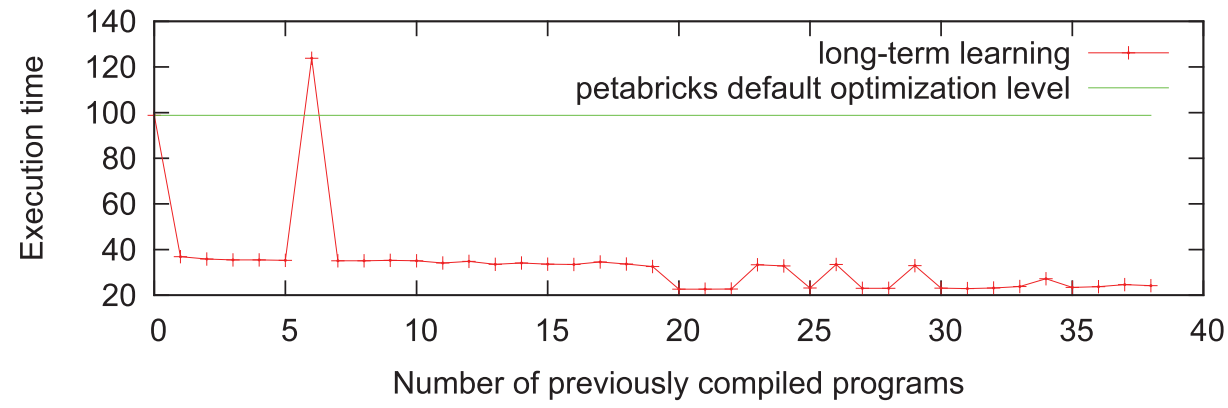

Fig. 15. Execution times of cholesky on Hardware Configuration 1 after the compilation of an increasing number of other programs. The speedup is computed with respect to PetaBricks default optimization level.

to find out the best configuration. This implies that every program version generated by our long-term learning algorithm has to be tuned before being run to measure its execution time and determine its speedup. Since the tuning process requires at least some minutes, the added delay with respect to the GCC implementation is not negligible.

PetaBricks programs are natively parallel, so they tend to occupy all the resources of the machine. In order to speed up the execution of the tests, we executed multiple tests in parallel using MapReduce as described in Tartara and Crespi Reghizzi [2012].

We show the running times of a test computing the Cholesky decomposition of a square matrix of size 256 (Figure 14). The default compiler configurations used as reference for the long-term learning algorithm are: optimizations disabled (activated by the -00 parameter in our modified PetaBricks version) and default (that is, maximum) PetaBricks optimization level (does not require any particular command line option).

The spikes appearing in the graph are due to the underlying autotuner. Being based upon a genetic algorithm, it is not assured to always converge to the same solution. Therefore, it is possible that some configurations generated by long-term learning turn out to be harder to tune than most others. We can see that after an initial slow descent phase, long-term learning is able to find a good configuration. Moreover, the performance level reached is better than that of the default optimization level of PetaBricks.

The same happens in Figure 15, when using a square matrix of size 1024. 


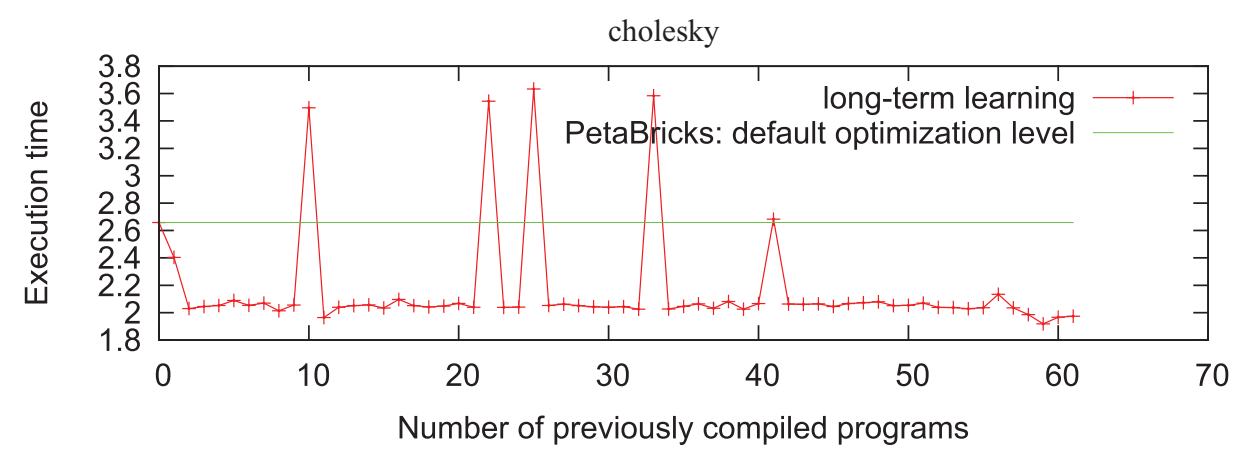

Fig. 16. Execution times of cholesky on Hardware Configuration 3 after the compilation of an increasing number of other programs. The speedup is computed with respect to PetaBricks default optimization level.

Figure 16 shows that a comparable reduction of the running times can be obtained on a different hardware configuration too.

\section{RELATED WORK}

Many researchers have worked towards building compilers without the need for compiler programmers to manually define whether to apply each optimization algorithm.

Agakov et al. [2006] show that it is possible to learn a model that predicts good optimizations based on the analysis of static program features. Experimental results from Cavazos et al. [2007] suggest that using performance counters instead of static features allows to further reduce the number of configurations to test.

Another approach was proposed by Dubach et al. [2007]. Every time they need to compile a program, they build a model by compiling and testing a small number (as low as 8) of versions of the program. Then, they use this model to predict-without execution - the performance of 500 randomly generated points of the search space. Up to 100 of the best points from this set are executed to find the actual best version. The problem with this approach is the need to build a program-specific model, and the fact that it does not reuse knowledge acquired from previous compilation.

Thomson et al. [2009] try to reduce the training time by focusing it on the programs that best characterize the search space of the optimization sets. They first gather the static features of all the programs in the training set, then apply unsupervised clustering in the program feature space. This way, a classification of the programs according to the similarity of their features is obtained. The most typical program of each cluster is chosen, obtaining a subset of programs that is representative of the whole search space. Each of the programs of the reduced training set is then compiled and tested with 4000 different optimizations sets, using a supervised learning technique to learn the model. The model is then used to immediately provide the supposedly best configuration, one-shot, without using iterative compilation.

The workflow proposed by Long [2011] uses a reverse $k$-nearest neighbors classifier to determine if the program being compiled is similar to a previously met one. If it is, the best configuration that was recorded for the similar program is used. If the program is an outlier, iterative compilation with random search is used to search for good configurations. Information about all the tested candidate configurations is stored. This approach is similar to long-term learning because it does not need a training phase and reduces the number of times iterative compilation has to be used. On the other hand, when it is used, hundreds of executions are needed.

The main differences between long-term learning and other approaches are as follows. Most works just aim at improving the performance of the compiled programs. 
Long-term learning, on the other hand, also aims at finding the best compiler heuristics expressed through human-readable formulas, that are easier to understand for compiler writers. To the best of our knowledge, the only other works with the same aim are those by Stephenson et al. [2003], using mathematical formulas, and Monsifrot et al. [2002], using decision trees. Furthermore, most works learn the single heuristics independently, whereas we also score sets as a whole, capturing the interactions between heuristics. Finally, most works just deal with changing command line flags, whereas our approach is more integrated in the compiler and can change internal parameters of the algorithms as well, as shown with PetaBricks tiling algorithm.

\section{FUTURE WORK}

Various directions for improvements are possible, and we mention just a few.

Sometimes, different compiler configurations applied to the same source file might lead to identical binaries [Kulkarni et al. 2004]. Moreover, since long-term learning is a multi-generation learning algorithm and uses elitism, it is likely that some candidates will be present, identical, in multiple generations. To prevent testing the same binary candidate more than once, we might compute a hash of the binary file, reusing previously recorded execution times where available. The default settings of our long-term learning implementation test a total of 18 candidates (as explained in Section 4.1) during a compilation. The timing runs are the slowest step of our algorithm, so, avoiding even just one of them would sensibly speed up the compilation process.

Currently, the selection of what program features to use in heuristic formulas is done randomly. A statistical approach computing the correlation between features used and the obtained speedup is likely to perform better. Also, adding the ability to compute more static program features, and the ability to use dynamic features derived from performance counters, might improve the quality of the heuristics.

Currently, long-term learning always tests a fixed number of candidate configurations. Although low, this number still might cause quite long compilation times. Using program features, we might determine whether many similar programs have been compiled before. We could then trust the current heuristics and just generate the best candidate accordingly (as in Long [2011]), or, if the program is completely different from anything known, the full set of 18 candidates should be tested. Intermediate configurations (for example with just 1 or 2 generations instead of 3 ) could also be used when only some data is available from previously compiled similar programs.

For this article, we decided to use GCC because, being at the heart of PetaBricks, we could share part of the implementation effort for the two compilers. LLVM [Lattner and Adve 2004] could be another interesting compiler on which to implement longterm learning, as it provides a wide variety of optimization passes and the possibility to write a pass manager to decide which ones to execute using our algorithm.

\section{CONCLUSIONS}

This article presented long-term learning, a novel algorithm to determine the best set of heuristics that a compiler can use to make decisions about which optimizations to enable and what values to assign to parameters, without any human intervention.

Experimental results on multiple architectures confirm that, when implemented in the GCC and PetaBricks compilers, the algorithm is able to obtain good results starting from no knowledge at all about good optimizations, reaching and sometimes surpassing the performance of the respective maximum optimization levels.

We also verified that when some initial knowledge is provided by the compiler writer, the algorithm is able to improve the performance, sometimes marginally, sometimes in a significant way. By analyzing more static and dynamic features we could improve 
the ability of the system to characterize the program being compiled, thus further increasing the quality of the result and the obtainable speedups.

Long-term learning improves the compiler by using the information it gathers from the execution time of the compiled programs. In order to compute it, it needs input data for the programs to be provided. Therefore, the ideal use of a long-term learning compiler is probably inside a test-driven development workflow, where the test data are prepared before the program itself and are already available during the compilation.

Long-term learning is meant to learn compiler heuristics, therefore, it is not strictly required for it to always be active. It allows the user to obtain compiler heuristics targeted at producing good programs for the architecture they will actually run on, not just good programs obtained by the iterations performed while the algorithm is active. Therefore, once satisfying heuristics have been learned, the long-term learning algorithm can be completely disabled, leaving a fast compiler producing optimized programs. Also, given two identical systems, the heuristics learned on one system can be ported on the other immediately giving good results on new programs.

\section{ACKNOWLEDGMENTS}

The authors would like to thank Jason Ansel, Professor Saman Amarasinghe and Professor Una-May O'Reilly, of MIT, and all the anonymous reviewers for their suggestions and valuable comments.

\section{REFERENCES}

Agakov, F. V., Bonilla, E. V., Cavazos, J., Franke, B., Fursin, G., et Al. 2006. Using machine learning to focus iterative optimization. In Proceedings of the International Symposium on Code Generation and Optimization. 295-305.

Ansel, J., Chan, C. P., Wong, Y. L., Olszewski, M., ZhaQ, Q., ET AL. 2009. Petabricks: A language and compiler for algorithm choice. In Proceedings of the 2009 ACM SIGPLAN Conference on Programming Language Design and Implementation. 38-49.

Ansel, J., Pacula, M., Amarasinghe, S. P., and O'Reilly, U. -M. 2011. An efficient evolutionary algorithm for solving incrementally structured problems. In Proceedings of the $13^{\text {th }}$ Annual Conference on Genetic and Evolutionary Computation. 1699-1706.

ARLANDINI, C. AND INVERNIZZI, A. 2008. Lagrange: Un nuovo server per il calcolo ad alte pretazioni. Bollettino del CILEA 0, 110.

Blum, A. 1996. On-Line algorithms in machine learning. In Proceedings of the Workshop on OnLine Algorithms. Springer, 306-325.

Bodin, F., Kisuki, T., Knijnenburg, P., O’Boyle, M., and Rohou, E. 1998. Iterative compilation in a non-linear optimization space. In Proceedings of the Workshop on Profile and Feedback Directed Compilation in conjunction with the International Conference on Parallel Architectures and Compilation Techniques (PACT).

Cavazos, J., Fursin, G., Agakov, F. V., Bonilla, E. V., O’Boyle, M. F. P., Et Al. 2007. Rapidly selecting good compiler optimizations using performance counters. In Proceedings of the $5^{\text {th }}$ Annual International Symposium on Code Generation and Optimization. 185-197.

Dubach, C., Cavazos, J., Franke, B., Fursin, G., O’Boyle, M. F. P., Et al. 2007. Fast compiler optimisation evaluation using code-feature based performance prediction. In Proceedings of the $4^{\text {th }}$ International Conference on Computing Frontiers. 131-142.

Dubach, C., Jones T. M., Bonilla E. V., Fursin, G., And O’Boyle, M. F. P. 2009 Portable compiler optimisation across embedded programs and microarchitectures using machine learning. In Proceedings of the $42^{\text {nd }}$ Annual IEEE/ACM International Symposium on Microarchitecture. 78-88.

Fogel, G. 2000. What is evolutionary computation? IEEE Spectrum 37, 2, 26-32.

Fursin, G. 2010. Collective benchmark (cbench), a collection of open-source programs with multiple datasets assembled by the community to enable realistic benchmarking and research on program and architecture optimization. http://ctuning.org/cbench

Fursin, G., Cavazos, J., O’Boyle, M. F. P., and Temam, O. 2007 Midatasets: Creating the conditions for a more realistic evaluation of iterative optimization. In Proceedings of the International Conference on High Performance Embedded Architectures \& Compilers. 245-260. 
Fursin, G., Kashnikov, Y., Memon, A. W., Chamski, Z., Teman, O., Et AL. 2011. Milepost gcc: Machine learning enabled self-tuning compiler. Int. J. Parallel Program 39, 3, 296-327.

Fursin, G., Miranda, C., Temam, O., Namolaru, M., Yom-Tov, E., Et al. 2008. Milepost gcc: Machine learning based research compiler. In Proceedings of the GCC Developers Summit.

Fursin, G. And Temam, O. 2010. Collective optimization: A practical collaborative approach. ACM Trans. Archit. Code Optim. 7, 4, 20.

Guthaus, M. R., Ringenberg, J. S., Ernst, D., Austin, T. M., Mudge, T., ET AL. 2001. Mibench: A free, commercially representative embedded benchmark suite. In Proceedings of the IEEE International Workshop on Workload Characterization (WWC'01). IEEE Computer Society, Los Alamitos, CA, 3-14.

Kaelbling, L. P., Littman, M. L., And Moore, A. W. 1996. Reinforcement learning: A survey. CoRR csAI/9605103.

Kisuki, T., Knisnenburg, P., O’Boyle, M., and WiJshoff, H. A. G. 2000. Iterative compilation in program optimization. http://citeseerx.ist.psu.edu/viewdoc/summary?doi=10.1.1.36.3007

KoHAVI, R. 1995. A study of cross- validation and bootstrap for accuracy estimation and model selection. In Proceedings of the $14^{\text {th }}$ International Joint Conference on Artificial Intelligence. 1137-1145.

Kulkarni, P. A., Hines, S., Hiser, J., Whalley, D. B., Davidson, J. W., ET AL. 2004. Fast searches for effective optimization phase sequences. In Proceedings of the ACM SIGPLAN 2004 Conference on Programming Language Design and Implementation. 171-182.

LATTNER, C. AND AdVE, V. S. 2004. LLVM: A compilation framework for lifelong program analysis \& transformation. In Proceedings of the International Symposium on Code Generation and Optimization. 75-88.

LEATHeR, H. 2011. Machine learning in compilers. Ph.D thesis, Institute of Computing Systems Architecture, School of Information, University of Edinburgh.

Leather, H., Bonilla, E. V., And O’Boyle, M. F. P. 2009. Automatic feature generation for machine learning based optimizing compilation. In Proceedings of the $7^{\text {th }}$ Annual IEEE/ACM International Symposium on Code Generation and Optimization. 81-91.

Long, S. 2011. Sustainable learning-based optimization based on rknm outlier detection. In Proceedings of the $5^{\text {th }}$ Workshop on Statistical and Machine Learning Approaches to Architecture and Compliation (SMART'11).

MerRILL, J. 2003. GENERIC and GIMPLE: A new tree representation for entire functions. In Proceeedings of the GCC Summit. Red Hat Inc.

Monsifrot, A., Bodin, F., AND Quiniou, R. 2002. A machine learning approach to automatic production of compiler heuristics. In Proceedings of the $10^{\text {th }}$ International Conference on Artificial Intelligence: Methodology, Systems, and Applications. 41-50.

Namolaru, M., Cohen, A., Fursin, G., Zaks, A., And Freund, A. 2010. Practical aggregation of semantical program properties for machine learning based optimization. In Proceedings of the International Conference on Compilers, Architectures and Synthesis for Embedded Systems. 197-206.

Park, E., Kulkarni, S., ANd Cavazos, J. 2011. An evaluation of different modeling techniques for iterative compilation. In Proceedings of the $14^{\text {th }}$ International Conference on Compilers, Architectures and Synthesis for Embedded Systems. 65-74.

Russell, S. J. and Norvig, P. 2010. Artificial Intelligence - A Modern Approach $3^{\text {rd }}$ ED. Pearson Education.

Stephenson, M., Amarasinghe, S. P., Martin, M. C., And O'Reilly, U.-M. 2003. Meta optimization: Improving compiler heuristics with machine learning. In Proceedings of the ACM SIGPLAN 2003 Conference on Programming Language Design and Implementation. 77-90.

Tartara, M. and CRespi Reghizzi, S. 2012. Parallel iterative compilation: Using MapReduce to speedup machine learning in compilers. In Proceedings of the $3^{\text {rd }}$ International Workshop on MapReduce and its Applications (MAPREDUCE'12).

Thomson, J., O'Boyle, M. F. P., Fursin, G., And Franke, B. 2009. Reducing training time in a one-shot machine learning-based compiler. In Proceedings of the $22^{\text {nd }}$ International Workshop on Languages and Compilers for Parallel Computing (LCPC'09). 399-407.

Received June 2012; revised November 2012; accepted November 2012 